Check for updates

Cite this: Food Funct., 2020, 11 10341

Received 28th August 2020 Accepted 18th September 2020 DOI: $10.1039 / \mathrm{dOfo} 02258 \mathrm{f}$

rsc.li/food-function

\section{Black bean protein concentrate ameliorates hepatic steatosis by decreasing lipogenesis and increasing fatty acid oxidation in rats fed a high fat-sucrose diet}

\author{
Irma Hernandez-Velazquez, $\uparrow^{\mathrm{a}}$ Monica Sanchez-Tapia, $\uparrow^{\mathrm{b}}$ Guillermo Ordaz-Nava, ${ }^{\mathrm{b}}$ \\ Nimbe Torres, (iD ${ }^{\mathrm{b}}$ Armando R. Tovar ${ }^{\mathrm{b}}$ and Amanda Galvez (iD *c
}

\begin{abstract}
The black bean is a legume widely consumed in Latin America, however its consumption has decreased significantly in recent decades. There is evidence that its consumption generates beneficial health effects due in part to the type of protein, resistant starches and polyphenols. Thus, their use in food formulation could impact health status. Therefore, the purpose of the present work was to evaluate the effects of the consumption of a bean protein concentrate (BPC) and a whole cooked bean flour (WCB) on body composition, glucose metabolism and energy expenditure in Wistar rats fed a control diet or high-fat diets with $5 \%$ sucrose in the drinking water. With this aim, rats were fed the experimental diets for 10 weeks. The results showed that consumption of either BPC or WCB reduced weight gain and body fat despite the consumption of a high-fat diet. This change was associated with a significant increase in energy expenditure and the capacity to adapt fuel oxidation to fuel availability. As a result, rats fed a bean-based diet had lower circulating glucose and insulin concentrations and normal glucose tolerance, which was associated with decreased expression of lipogenic genes in the liver. These results suggest that the type of protein and bioactive compounds particularly phenolic and flavonoid compounds present in BPC are suitable to improve the formulations used in dietary strategies for subjects with obesity or type 2 diabetes. The addition of legumes to the diet of subjects with insulin resistance, including black beans, could improve their metabolic status.
\end{abstract}

\section{Introduction}

Legumes show low energy density and are nutrient dense foods associated with good health. ${ }^{1}$ In Mesoamerica, beans (Phaseolus vulgaris), are the staple food of legumes that have lost their former primary place as a source of protein in diets, perhaps due to their association with low-income groups. Different varieties of beans show protein contents varying between 17 and 23\%, approximately 15\% dietary fiber, 60\% starch content ${ }^{2-6}$ and amounts varying between 3 and $5 \mathrm{~g}$ of resistant starch per $100 \mathrm{~g}$ after 60 min of cooking. ${ }^{3,7,8}$ In addition, beans contain flavonoids and polyphenols that give them antioxidant properties, ${ }^{9,10}$ depending on the particular genotype, the location of crop growth, and the effect of

${ }^{a}$ Comision Nacional para el Uso y Conocimiento de la Biodiversidad. Coordinacion de Agrobiodiversidad, Mexico

${ }^{b}$ Instituto Nacional de Ciencias Médicas y Nutrición Salvador Zubirán, Mexico ${ }^{c}$ Dept. Alimentos y Biotecnología. Facultad de Química, Universidad Nacional Autónoma de México, Mexico.E-mail: galvez@unam.mx

$\dagger$ These authors contributed equally to this work. environmental factors such as storage conditions and time. A meta-analysis of general legume consumption has concluded that a high consumption of legumes is associated with a lower risk of all-cause mortality; however, the number of clinical studies is currently limited. ${ }^{11}$ In addition, one clinical trial showed that consumption of black beans, when included in a typical western-style meal, decreases postprandial insulin and moderately improves postprandial antioxidant endpoints in adults with metabolic syndrome, in part because of their fiber content and antioxidant capacity. ${ }^{12,13}$ However, the biological effects and the molecular mechanism by which black bean protein regulates carbohydrate and lipid metabolism have not been investigated in detail. The comorbidities of obesity have been associated with the development of metabolic inflexibility, a condition that occurs when mitochondria are not able to switch adequately between different energy substrates, particularly between glucose and fatty acids. ${ }^{14,15}$ This can lead to insulin resistance and lipotoxicity. According to Rebello et al., 2014 , the addition of legumes to the diet of subjects with insulin resistance, including black beans, could improve their metabolic status. ${ }^{1}$ 
Currently, the demand for new sources of plant protein is increasing in light of current trends in vegetarianism and veganism. In addition, patients with type 2 diabetes mellitus, or who are developing a metabolic syndrome, may also benefit from the inclusion of plant protein materials in food formulation $^{16}$ because of this capacity to reduce postprandial glucose and insulin peaks.

The objectives of this study were to study the nutritional effects of two bean preparations, whole cooked bean flour (WCB) and a black bean protein concentrate (BPC), in Wistar rats fed for 10 weeks adequate or high-fat diets with $5 \%$ of sucrose in the drinking water, on body composition, energy expenditure and glucose and lipid metabolism, compared with those of rats fed appropriate or high-fat diets containing casein according to AIN93, which has been used as the reference protein. It is important to note that the bean protein concentrate (BPC) was stripped of most of its starch fractions to have a high protein content (63\% protein), which showed good functional properties (good emulsification capacity, water and oil absorption capacity), as well as an acceptable solubility. Thus, BPC is a good protein extension ingredient containing dietary bioactive compounds mainly polyphenols and flavonoids, especially for processed cereal-based foods, in order to supplement, as well as increase, their protein content (Patent dossier MX/a/2015/007746). On the other hand, whole cooked beans flour (WCB), prepared by drum drying, preserved all the carbohydrate fractions of the beans.

\section{Materials and methods}

\subsection{Black bean protein concentrate}

A protein concentrate was obtained from black beans of the Bola variety by differential solubility extraction of all protein fractions followed by isoelectric precipitation, heat treatment at $121{ }^{\circ} \mathrm{C}$ for $80 \mathrm{~min}$ for the inactivation of antinutritional factors, and spray-drying. The concentration process was performed at the Institute of Biotechnology Pilot Plant (IBT-UNAM), according to the patent recently granted by the Mexican Institute of Industrial Protection, IMPI (Patent dossier no. MX/a/2015/007746).

\subsection{Dehydrated whole cooked black bean}

A $100 \mathrm{~kg}$ batch of whole Bola black beans was cooked and dehydrated in a rotating drum dryer at the company Defrut (Morelia, Michoacán, Mexico), using their commercial thermal process that allowed proper deactivation of antinutritional factors (refer to the data below). This material is hereby named whole cooked bean (WCB).

\subsection{Trypsin inhibitor and starch content determinations}

Trypsin inhibitor units (TIU) were measured according to Kakade in 1974 and Klomklao et al., 2011 with small modifications. ${ }^{17,18}$ Total starch (TS) and resistant starch (RS) contents were also determined using Megazyme kits: total starch kit (Ireland, UK).

\subsection{Experimental design and dietary treatment}

Six-week-old male Wistar rats were randomly assigned to six groups, $n=6$ in each group: (1) casein (C) (control group); (2) black bean protein concentrate (BPC); (3) whole cooked bean (WCB); (4) a casein high fat $+5 \%$ sucrose in the drinking water (HFS), (C + HFS); (3) BPC + HFS; (4) WCB + HFS (Fig. 1). This animal protocol was approved by the Institutional Animal Care and Research Advisory Committee of the Instituto Nacional de Ciencias Médicas y Nutrición Salvador Zubirán, Mexico City (CINVA 1744). All animals were fed for 10 weeks ad libitum with free access to water. Diets were formulated according to the recommendations of AIN-93. ${ }^{19}$ In Table 1, composition and energy of diets is reported. Body weight and food intake were registered three times per week. The volume of $5 \%$ sucrose water consumed was also measured in order to determine the energy intake. At week 10, rats were euthanized by decapitation after being anesthetized with $\mathrm{CO}_{2}$. The liver and ileum were removed immediately, frozen in liquid nitrogen, and stored at $-70{ }^{\circ} \mathrm{C}$ until analysis, and serum was obtained by centrifugation of blood at $1500 \mathrm{~g}$ for $10 \mathrm{~min}$ and stored at $-70{ }^{\circ} \mathrm{C}$.

\subsection{Body composition}

The body composition of all animals was measured at the beginning of the experiment and at days 15, 30, 45, 60 and 70 of experimental diet consumption using the ECHO-MRI quantitative magnetic resonance equipment for small animals (Echo Medical Systems, Houston, TX). This methodology

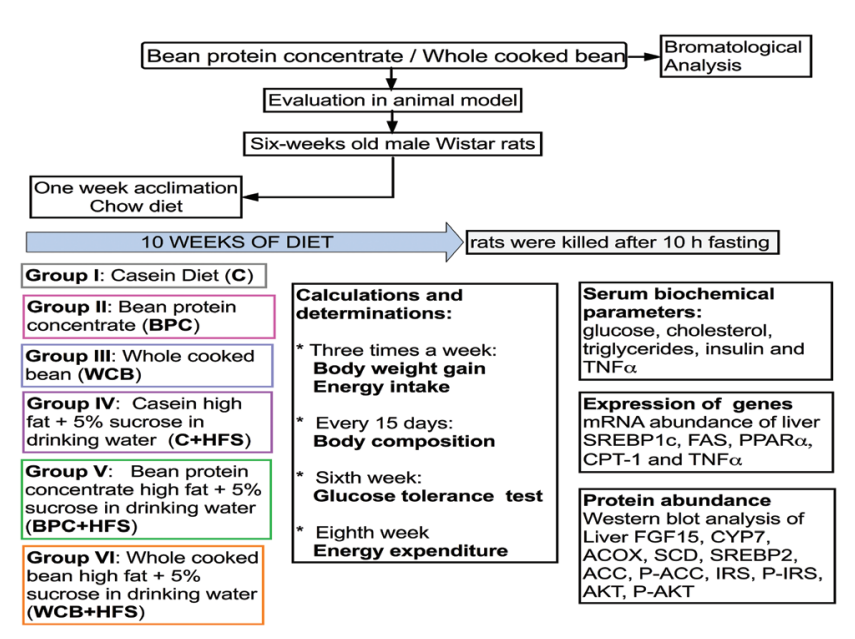

Fig. 1 Study design.

Table 1 Energy and macronutrient content in the diets (g per $100 \mathrm{~g}$ )

\begin{tabular}{lcrrlll}
\hline Nutrient & C & BPC & WCB & C + HFS & BPC + HFS & WCB + HFS \\
\hline Carbohydrates & 66.0 & 66.3 & 66.3 & 42.1 & 42.5 & 42.5 \\
Lipids & 7.0 & 7.0 & 7.0 & 24.0 & 23.9 & 23.9 \\
Protein & 17.0 & 16.9 & 16.9 & 24.0 & 23.9 & 23.9 \\
kcal g $^{-1}$ diet & 3.95 & 3.96 & 3.96 & 4.80 & 4.81 & 4.81
\end{tabular}


allowed to obtain the fat and lean mass, and the water content of each experimental animal.

\subsection{Energy expenditure}

At week 8 of experimental diet consumption, all rats were placed individually in a computer-controlled open-circuit indirect respiration calorimeter (Oxymax instrument, CLAMS, Columbus Instruments, Columbus, $\mathrm{OH}$ ) to obtain the volume of $\mathrm{O}_{2}$ consumption $\left(\mathrm{VO}_{2}\right)$ and $\mathrm{CO}_{2}$ production $\left(\mathrm{VCO}_{2}\right)$. The energy expenditure was expressed as heat $\left(\mathrm{kcal} \mathrm{kg}^{-1} \mathrm{~h}^{-1}\right)$ as previously described. ${ }^{20}$ Heat is derived by assessment of the exchange of oxygen for carbon dioxide that occurs during the metabolic process.

\subsection{Serum biochemical parameters}

At the end of the experiment, blood was collected in tubes with clot activator and gel (BD Vacutainer, Franklin Lakes NJ, USA) and centrifuged at $1000 \mathrm{~g}$ for $15 \mathrm{~min}$. Samples were stored at $-70{ }^{\circ} \mathrm{C}$ until further analysis. Glucose, cholesterol and triglycerides (TG), were measured with a Cobas c111 analyzer (Roche) based on enzymatic-photometric assays. Serum tumor necrosis factor- $\alpha(\mathrm{TNF} \alpha)$ was measured by ELISA (Alpco, 61-TNART-E01).

\subsection{Glucose tolerance test}

A glucose tolerance test was performed in all rats fasted for 8 hours after 6 weeks of the dietary treatment as previously described. ${ }^{21}$ The intraperitoneal glucose tolerance test was carried out by administering an intraperitoneal glucose injection of $2 \mathrm{~g}$ per $\mathrm{kg}$ body weight in rats after an 8-hour fast. Blood samples were collected from the tail vein at $0,15,30,45$, 60,90 , and $120 \mathrm{~min}$ after administration of the glucose. Blood glucose concentration was measured using a FreeStyle Optium glucometer (Abbot Laboratories, Abbot Park, IL, USA). The area under the curve was determined by the trapezoid method.

\subsection{Liver cholesterol and triglyceride concentrations}

Liver lipids were extracted from $100 \mathrm{mg}$ of tissue using chloroform-methanol, according to the method described by Folch et $a l^{22}$ The organic layer was dried using liquid nitrogen and solubilized in isopropanol/Triton X-100 (10\%). The concentrations of total cholesterol and triglycerides were measured with an enzymatic colorimetric kit (DiaSys Diagnostic Systems, Germany).

\subsection{Histological analysis}

Liver sections of $4 \mu \mathrm{m}$ thick were stained with hematoxylin and eosin. A morphological analysis was performed using a Leika microscope (Leika DM750 Wetzlar, Germany).

\subsection{Gene expression analysis}

Total RNA was extracted from liver according to the method reported by Chomczynski and Sacchi. ${ }^{23}$ The synthesis of cDNA was performed using an M-MLV reverse transcriptase enzyme (Invitrogen, Carlsbad, CA). mRNA abundance of sterol regulatory element-binding protein (SREBP 1c), fatty acid synthase
(FAS), peroxisome proliferator-activated receptor (PPAR $\alpha$ ), carnitine palmitoyl transferase-1 (CPT-1) and tumor necrosis factor $(\mathrm{TNF} \alpha)$ were measured by real-time quantitative PCR using the SYBR Green I Master assay (Roche Applied Science, Germany) in triplicate, using a LightCycler 480 Instrument (Roche Applied Science, Germany). The PCR scheme used was $50{ }^{\circ} \mathrm{C}$ for $2 \mathrm{~min}, 95^{\circ} \mathrm{C}$ for $10 \mathrm{~min}$, and 45 cycles of $94^{\circ} \mathrm{C}$ for 15 $\mathrm{s}$ followed by $64^{\circ} \mathrm{C}$ for $1 \mathrm{~min}$. Cyclophilin and HPRT were used as univariate endogenous controls. The primers used are shown in Table 2. The relative amount of mRNA was calculated using the $\Delta \Delta$ Ct method with an efficiency adjustment according to the Pfaffl equation. ${ }^{24}$

\subsection{Western blot analysis}

Total protein from liver samples were extracted and quantified by Bradford assay (Bio-Rad, Hercules, CA, USA) and stored at $-70{ }^{\circ} \mathrm{C}$. Protein samples were separated on $10 \%$ acrylamide gels with $30 \mu \mathrm{g}$ protein per lane and blotted onto PVDF membranes. Membranes were blocked in a blocking buffer consisting of $3 \% \mathrm{BSA}$ in TBS tween for $60 \mathrm{~min}$ at room temperature and incubated overnight at $4{ }^{\circ} \mathrm{C}$ with the primary antibody with the following primary and secondary antibodies in blocking buffer: anti-IRS1 (Santa Cruz, SC-560, rabbit polyclonal IgG) at $1: 750$, anti-pIRS1 (Santa Cruz, SC-17195-R, rabbit polyclonal IgG) at $1: 1000$, anti-AKT (Santa Cruz, SC-83122, rabbit polyclonal) at $1: 2500$, anti-pAKT (Santa Cruz, SC-7985, rabbit polyclonal) at $1: 5000$, anti-ACC (Santa Cruz, SC-30212, rabbit polyclonal) at $1: 1000$, anti-pACC (Millipore, 07-303, rabbit polyclonal) at $1: 2500$, anti-SCD1 (Santa Cruz, SC-30081, rabbit polyclonal) at $1: 1000$, anti-SREBP-2 (Santa Cruz, SC-8151, gout polyclonal) at 1:1000, anti-ACOX-1 (Santa Cruz, SC-98499, rabbit polyclonal) at 1:1000, anti-FGF15 (Santa Cruz, SC-16817, gout polyclonal) at 1:1000, anti-CYP7A1 (Abcam, AB65596, rabbit polyclonal) at $1: 3500$. The blots were incubated with anti-rabbit (Abcam AB6721) or anti-gout (Abcam AB6885) secondary antibodies conjugated with horseradish peroxidase $(1: 20000)$. GAPDH (Abcam, AB181602, rabbit monoclonal) at 1:20000 was used to normalize the data. Images were analyzed with a ChemiDocTM XRS + System Image LabTM Software (Bio-Rad, Hercules, CA, USA), and den-

Table 2 Primer sequences used for RT-qPCR

\begin{tabular}{lll}
\hline Gene & Primer & Sequence $\left(5^{\prime}-3^{\prime}\right)$ \\
\hline HPRT & Forward & CTGGTGAAAAGGACCTCTCG \\
& Reverse & GGCCACATCAACAGGACTCT \\
Cyclophilin & Forward & TGACTTTAGGTCCC \\
& Reverse & TTCTTCTTATCG \\
FREBP $1-c$ & Forward & CGTTGTACTGCAGC \\
& Reverse & CGTTGTACTGCAGCCACACT \\
FAS & Forward & AGTGGTACTGTGGCCAGGAT \\
& Reverse & CGGCGAGTCTATGCCACT \\
PPAR $\alpha$ & Forward & GCGTACGACAAGTGTGATCG \\
& Reverse & GCCAGAGATTTGAGGTCTGC \\
FPT-1 & Forward & GGATCCACCATTCCACTCTG \\
& Reverse & TCGTGGTAGAGCCAGACCTT \\
$T N F-\alpha$ & Forward & ATGTGGAACTGGCAGAGGAG \\
& Reverse & GCCATGGAACTGAGAGAGG
\end{tabular}


sitometric analysis was performed using the Image software (Version 1.51k). The assays were performed three times using independent blots.

\subsection{Statistical analyses}

Values are expressed as mean \pm standard error of the mean (SEM). Data were analyzed using GraphPad Prism (version 5.0, Graph Pad Software, Inc. La Jolla, CA, USA). Comparisons between groups were analyzed by a two-way ANOVA followed by a Tukey's post hoc test $(p<0.05)$.

\section{Results}

\subsection{Protein and trypsin inhibitor in BPC and WCB}

The BPC used for the experimental diets contained $63.3 \mathrm{~g}$ per $100 \mathrm{~g}$ of protein, while the WCB contained $17.8 \mathrm{~g}$ per $100 \mathrm{~g}$, respectively. The fiber content in the BPC was $3.2 \mathrm{~g}$ per $100 \mathrm{~g}$ and for WCB was $17.0 \mathrm{~g}$ per $100 \mathrm{~g}$. The heat treatment resulted in $31.01 \pm 1.81$ and $20.87 \pm 1.82$ trypsin inhibitor units (TIU per mg sample) for BPC and WCB, respectively. The values of TIU were in the normal range, considering 31 TIU as the maximum acceptable value.

\subsection{Consumption of BPC reduced body weight gain, body fat and increased energy expenditure}

Rats fed BPC for 10 weeks gained significantly less body weight than the control group, however, there was no signifi- cant difference in the \% of body fat and body lean mass with respect to the $\mathrm{C}$ group. Interestingly, rats fed $\mathrm{BPC}+\mathrm{HFS}$ gained 33.6\% less body weight gain than those fed $\mathrm{C}+\mathrm{HFS}$, and this was accompanied by a reduction of $11 \%$ of body fat and an increase of $5.5 \%$ of body lean mass in the BPC + HFS compared to the $\mathrm{C}+\mathrm{HFS}$. It is important to note that rats fed WCB + HFS showed even lower \% of body fat and higher \% of lean body mass than the group fed BPC + HFS $(p<0.05)$ (Fig. 2A-C). The results showed that the difference in body weight gain, $\%$ of body fat and \% of body lean mass were not due to difference in energy intake (Fig. 2D). Surprisingly, we observed that the groups fed either BPC + HFS or WCB + HFS showed significantly higher energy expenditure, particularly during the postprandial period in comparison to the $\mathrm{C}+\mathrm{HFS}$ group (Fig. 2E), suggesting that black bean stimulates energy expenditure even in the presence of a high-fat diet leading to lower body weight and body fat gain.

\subsection{Consumption of BPC reduced serum lipids, glucose and insulin, and improved glucose tolerance}

Rats fed WCB showed a reduction in the circulating concentrations of triglyceride and total cholesterol compared to the $\mathrm{C}$ group. However, after 10 weeks of dietary treatment with BPC + HFS or WCB + HFS, there was a significant reduction in serum triglycerides of $13.8 \%$ and $43.5 \%$ respectively compared to the $\mathrm{C}+$ HFS group. Similarly, BPC + HFS or WCB + HFS groups had a reduction of total cholesterol of $29.6 \%$ and $23.4 \%$ respectively compared to the $\mathrm{C}+\mathrm{HFS}$ group (Fig. $3 \mathrm{~A}$ and $\mathrm{B}$ ).
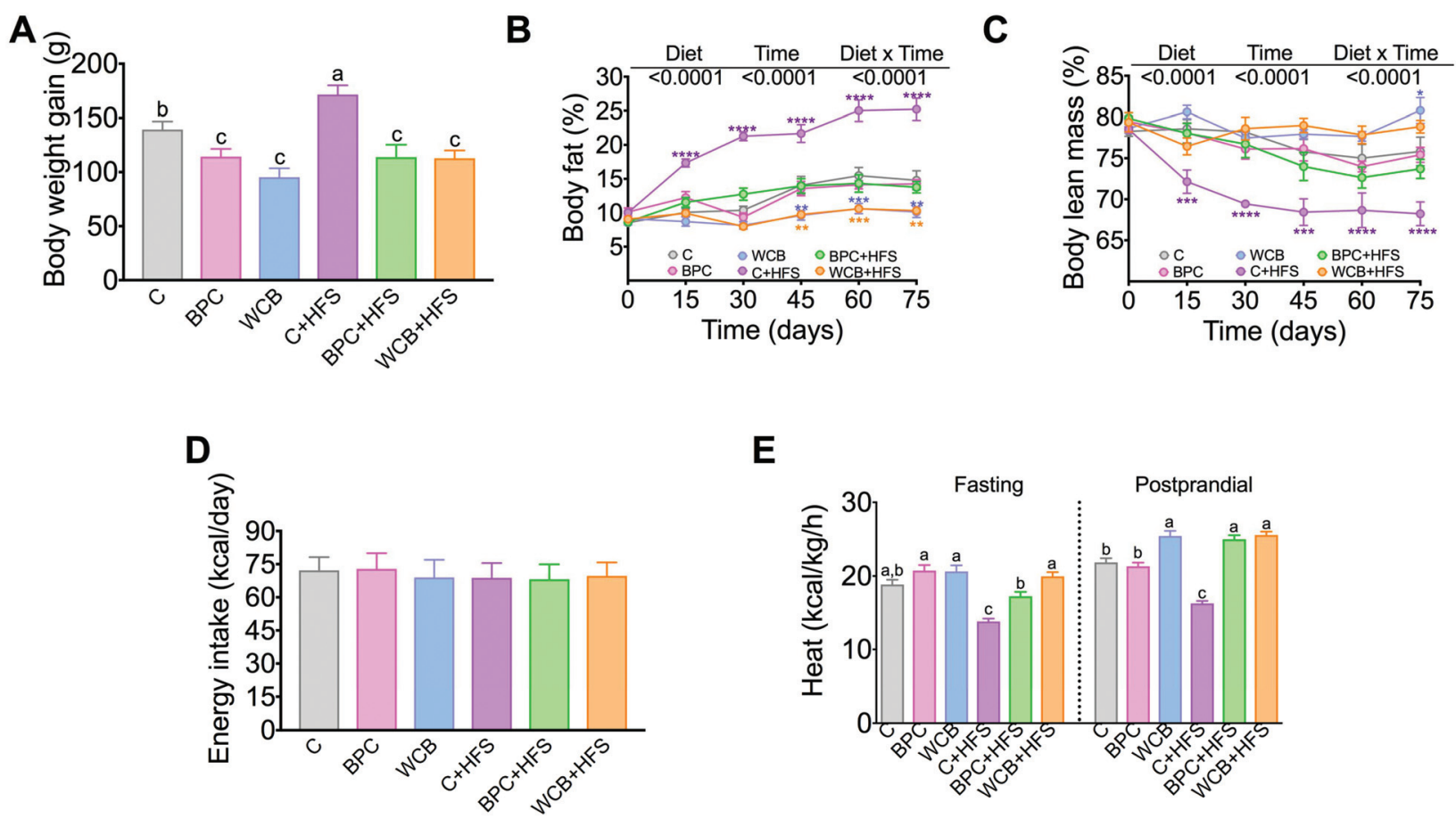

Fig. 2 Effect of black bean protein concentrate and whole cooked black bean on body weight gain, body composition and energy expenditure in rats fed 10 weeks the dietary treatment. (A) Body weight gain, (B) \% body fat, (C) \% body lean mass, (D) energy intake, (E) energy expenditure as heat. Data are mean \pm SEM, $n=6-7$ rats per group. Different letters indicate significant differences among groups, a $>b>c$; ${ }^{*}$ means a significant difference from group $C,(p<0.05)$. 
A
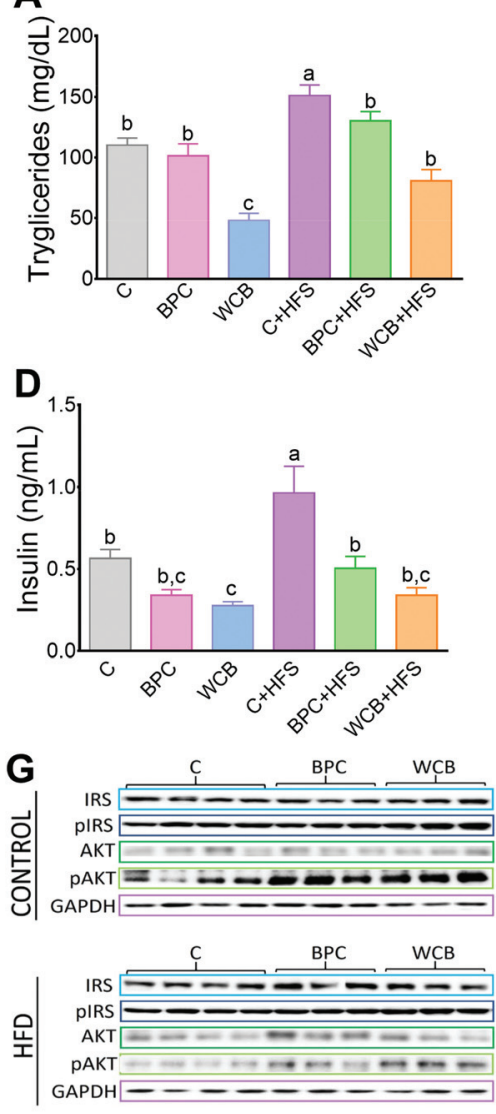

B

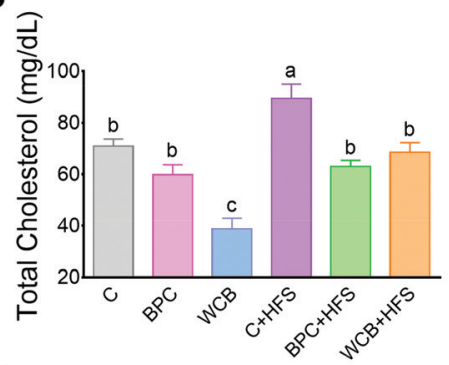

E

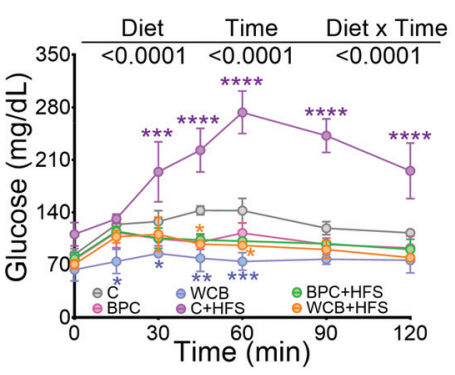

H

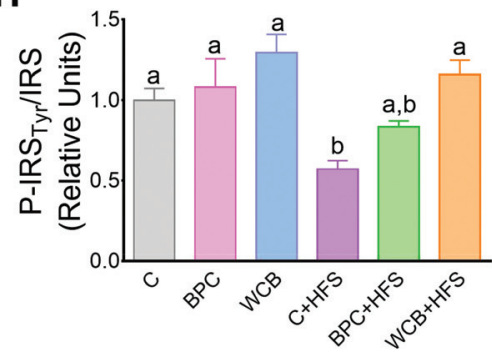

C
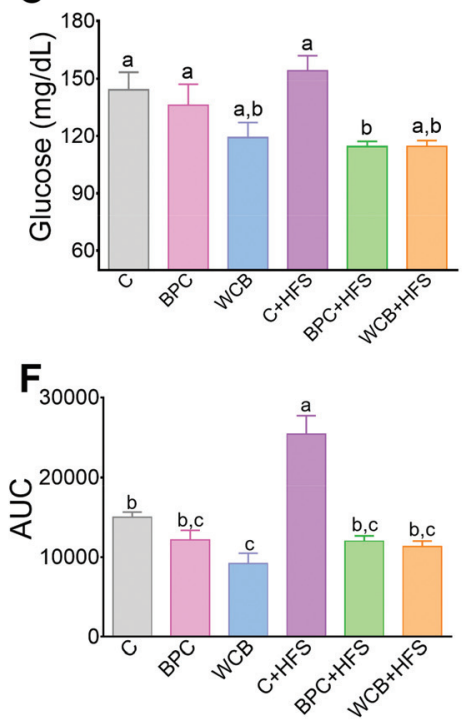

I

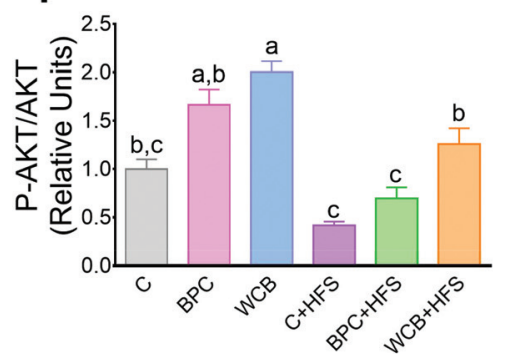

Fig. 3 Effect of black bean protein concentrate and whole cooked black bean on serum lipids, glucose and insulin, glucose tolerance test and protein abundance of enzymes of insulin signaling in rats fed for 10 weeks the dietary treatment. (A) Serum triglycerides, (B) serum total cholesterol, (C) serum glucose, (D) serum insulin, (E) glucose tolerance test, (F) area under the curve. (G) Western blot analyses of IRS-1, pIRS1, AKT and pAKT in liver, and $(\mathrm{H}$ and $\mathrm{I})$ densitometric analysis. Data are mean $\pm \mathrm{SEM}, n=6-7$ rats per group. Different letters indicate significant differences among groups, $a>b>c$; *means a significant difference from group $C,(p<0.05)$.

The results also showed that consumption of BPC or WCB improved fasting serum glucose and insulin concentrations, particularly in the groups fed high fat diets. Thus, the group fed BPC + HFS showed a significant decrease of $25.7 \%$ and $47.7 \%$ of serum glucose and insulin, respectively, compared to the C + HFS group. Similarly, the WCB + HFS had a reduction in serum glucose and insulin of $25.5 \%$ and $64.8 \%$, respectively compared to the C + HFS group (Fig. 3C and D). The improvement in carbohydrate metabolism by the consumption of black bean was evident after the glucose tolerance test. Rats fed C + HFS reached values of $273 \mathrm{mg} \mathrm{dl}^{-1}$ of serum glucose after $60 \mathrm{~min}$ of the administration of glucose, whereas rats fed $\mathrm{BPC}+\mathrm{HFS}$ or WCB + HFS, showed the maximal increase in blood glucose after $30 \mathrm{~min}$, reaching values between $115-130 \mathrm{mg} \mathrm{dl}^{-1}$, (Fig. 3E). In fact, the area under the curve (AUC), for those fed BPC + HFS or WCB + HFS showed a decrease of $52.7 \%$ and $55.5 \%$ respectively in comparison to the C + HFS group (Fig. 3F). The improvement in glucose tolerance particularly in the groups fed BPC + HFS or WCB + HFS was accompanied with an increase in the phosphorylation of p-IRS Tyr and p-AKT in liver, suggesting an improvement of insulin sensitivity with the consumption of black bean, (Fig. 3G-I).

\subsection{Resistant starch and flavonoid content in BPC and WCB}

The content of resistant starch in WCB was approximately 8.6 times higher than in BPC (Table 3). Total starch in BPC was also reduced by approximately $80 \%$, indicating that the process used for the production of PCB was effective in removing total and resistant starch. With respect to phenolic content, BPC showed a decrease of $23 \%$ and an increase of $98 \%$ in flavonoid content in comparison with WCB.

Table 3 Resistant starch, phenolic and flavonoids content in the WCB and BPC

\begin{tabular}{lll}
\hline COMPONENTS & WCB & BPC \\
\hline Resistant starch (g per 100 g sample) & $4.65 \pm 0.009$ & $0.483 \pm 0.05$ \\
Total starch (g per 100 g sample) & $51.24-56.76$ & 11.01 \\
Phenolics (mg gallic ac. per g sample) & $2.42 \pm 0.08$ & $1.85 \pm 0.08$ \\
Flavonoids (mg quercetin per g sample) & $0.96 \pm 0.04$ & $1.91 \pm 0.04$
\end{tabular}


3.5 Consumption of BPC reduced the accumulation of triglycerides in liver by modulating the expression of genes of lipogenesis and fatty acid oxidation

There is evidence that an increase in insulin sensitivity is associated with a lower accumulation of lipids in the liver. The results of the present study clearly showed that consumption of BPC or WCB significantly reduced the hepatic concentration of triglycerides (Fig. 4A). The histological analysis revealed, particularly in the groups fed high fat diets that either BPC or WCB reduced the hepatic steatosis, compared to the group fed $\mathrm{C}+$ HFS (Fig. 4B). It is noteworthy, that this effect was also observed in the groups fed BPC + HFS or WCB + HFS, where there was a reduction in hepatic triglycerides by $30.1 \%$ and $40.9 \%$ respectively, compared to the C + HFS group (Fig. 4A). Then, we explored whether the lower accumulation of triglycerides was associated with a reduction in the expression of lipogenic genes or an increase in the expression of genes associated with fatty acid oxidation. Therefore, we measured the abundance of mRNA of the transcription factor SREBP-1c and one of its target genes, FAS. Interestingly, the consumption of the $\mathrm{C}+$ HFS diet significantly increased the abundance of both SREBP-1c and FAS by 2- and 5.7-fold respectively compared to the $\mathrm{C}$ group. Consumption of BPC + HFS significantly decreased SREBP-1 and FAS by $63 \%$ and $80 \%$, respectively, whereas consumption of WCB + HFS reduced the abundance of both genes by $42 \%$ and $57 \%$, respectively, compared to $\mathrm{C}+$ HFS group (Fig. 4C and D). This was accompanied by inactivation of ACC by increasing its phosphorylation, that limits lipogenesis (Fig. 4E). Furthermore, we observed that consumption of BPC or WCB decreased the protein abundance of SCD (Fig. 4F), enzyme that catalyzes a rate-limiting step in the synthesis of unsaturated fatty acids. Therefore, consumption of black bean reduces the synthesis of saturated and monounsaturated fatty acids. On the contrary, when we studied genes involved in fatty acid oxidation, particularly PPAR $\alpha$ and CPT-1, we observed a significant increase in the expression of these genes after the consumption of black bean. The group fed WCB increased the mRNA abundance of PPAR $\alpha$ and CPT-1 by $97 \%$ and $54 \%$ respectively, compared to the $\mathrm{C}$ group. Consumption of $\mathrm{C}+$ HFS diet reduced the expression of both genes by $58 \%$ and $65 \%$ respectively, compared to the $\mathrm{C}$ group.
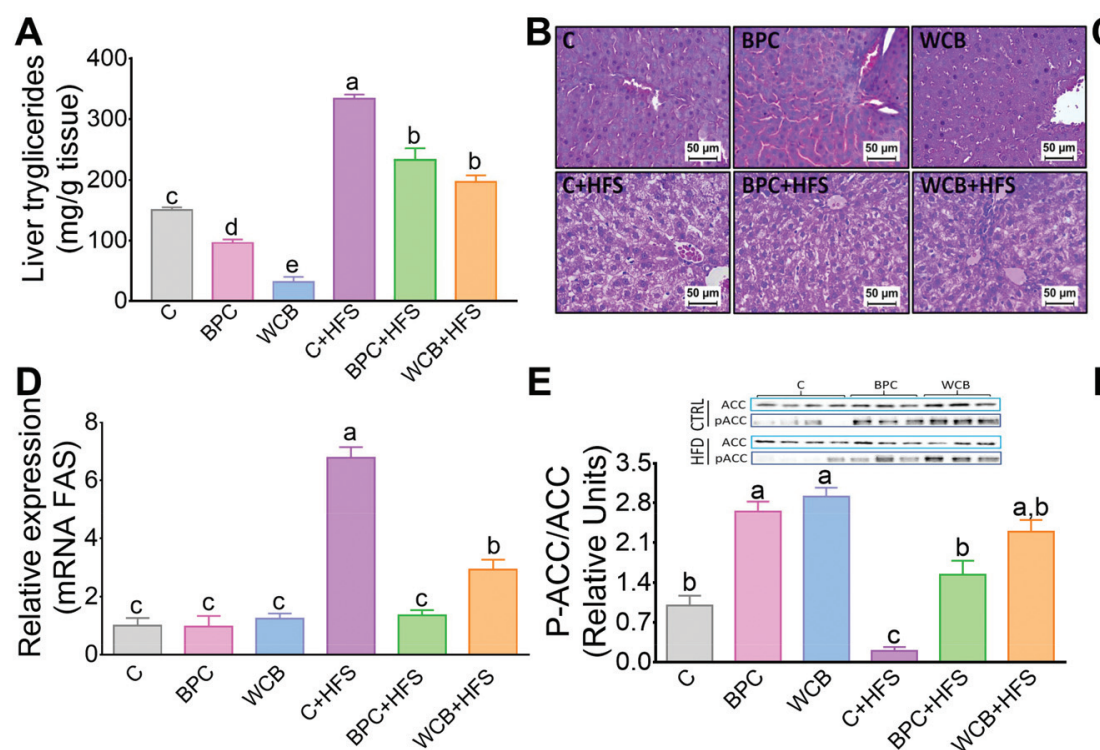

E

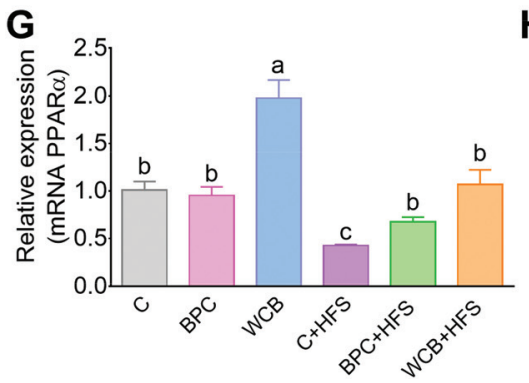

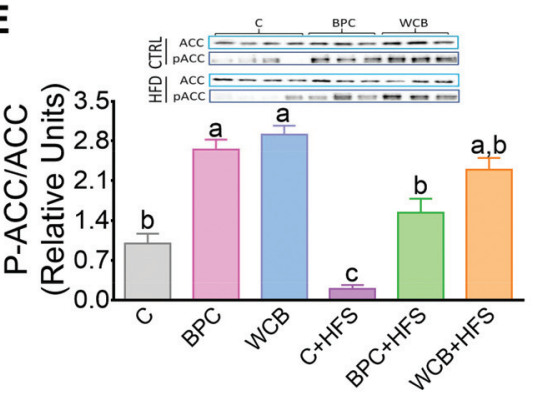

H

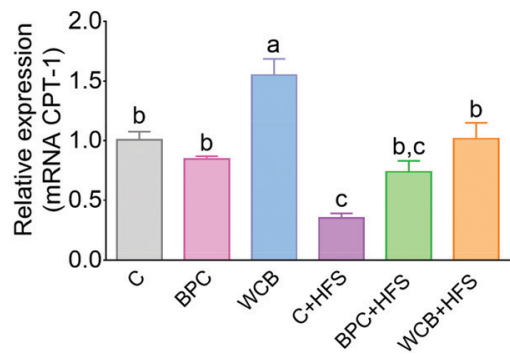

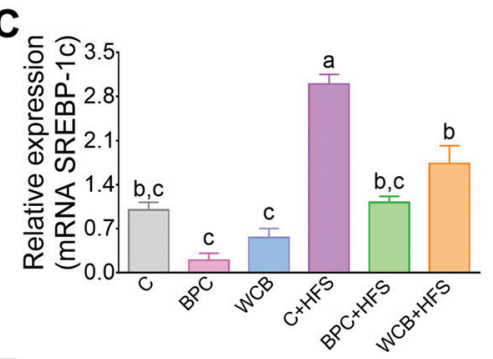

$\mathbf{F}$

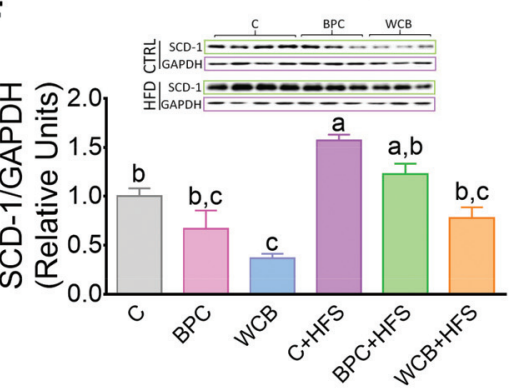

I

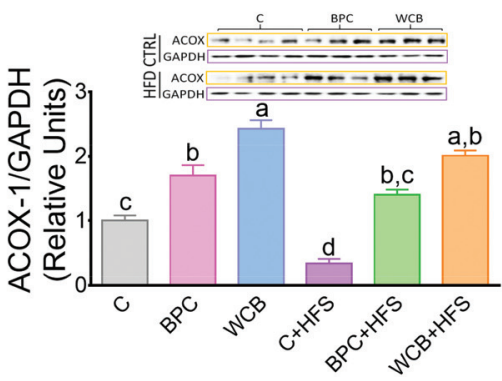

Fig. 4 Effect of black bean protein concentrate and whole cooked black bean on liver triglycerides, and the expression of lipogenic and fatty oxidation genes in rats fed black bean for 10 weeks. (A) Hepatic triglycerides, (B) liver histological analysis, (C) liver sterol regulatory element binding protein-1 (SREBP1), (D) fatty acid synthase (FAS) mRNA abundance, (E and F) western blot analysis and densitometry of acyl-CoA carboxylase (ACC), p-ACC, steoryl-CoA desaturase (SCD), (G) peroxisome proliferator-activated receptor (PPAR $\alpha$ ) mRNA abundance, (H) carnitine palmitoyltransferase-1 (CPT-1) mRNA abundance, (I) acyl CoA oxidase 1 (ACOX). Different letters indicate significant differences among groups, a $>b>c>d>e, p<=0.05$. 
However, the group fed BPC + HFS increased the mRNA abundance of PPAR $\alpha$ and CPT- 1 by $58 \%$ and $110 \%$ with respect to the $\mathrm{C}+$ HFS group. Similarly, consumption of $\mathrm{WCB}+\mathrm{HFS}$ increased the PPAR $\alpha$ and CPT- 1 mRNA abundance by 1.5- and 1.9-fold, respectively compared to the $\mathrm{C}+\mathrm{HFS}$ group (Fig. 4G and $\mathrm{H}$ ). In addition, consumption of black bean with or without a high fat diet increased the protein abundance of ACOX1, enzyme also involved in fatty acid oxidation (Fig. 4).

\subsection{Consumption of BPC reduced hepatic cholesterol and modules the expression of genes associated with cholesterol and bile acid synthesis}

Consumption of WCB also significantly reduced the hepatic concentration of cholesterol by $78.4 \%$ compared to the $\mathrm{C}$ group. These effects were also observed in the groups fed high fat diets where rats fed BPC + HFS or WCB + HFS decreased liver cholesterol concentration by $25.4 \%$ and $46.6 \%$ respectively with respect to the group fed $\mathrm{C}+\mathrm{HFS}$ (Fig. 5A). This reduction was accompanied by a significant decrease in the protein abundance of the transcription factor SREBP-2, involved in cholesterol synthesis (Fig. 5B and C).

As a consequence, the decrease in hepatic cholesterol after the consumption of black bean was associated with a reduction in bile acid synthesis, since the protein abundance of the rate limiting enzyme of the classic pathway of bile acid synthesis, CYP7A1 decreased with BPC and WCB (Fig. 5D and E). This mechanism was reinforced by an increase in the protein abundance of FGF15 in the ileum (Fig. 5D and F). FGF15 is known to repress the expression of Cyp7A1 via the FGF4 R/ $\beta$-Klotho receptor. ${ }^{25}$

\subsection{Consumption of black bean reduce the pro-inflammatory cytokine TNF $\alpha$}

There is evidence that TNF- $\alpha$ appears to play a key role in the development of hepatic steatosis. TNF- $\alpha$ is a proinflammatory cytokine that mediates hepatic inflammation. ${ }^{26}$ In fact, our results showed that rats fed $\mathrm{HF}+\mathrm{S}$ diet had a 1.5-fold increase in the hepatic mRNA abundance of TNF- $\alpha$ with respect to the
A
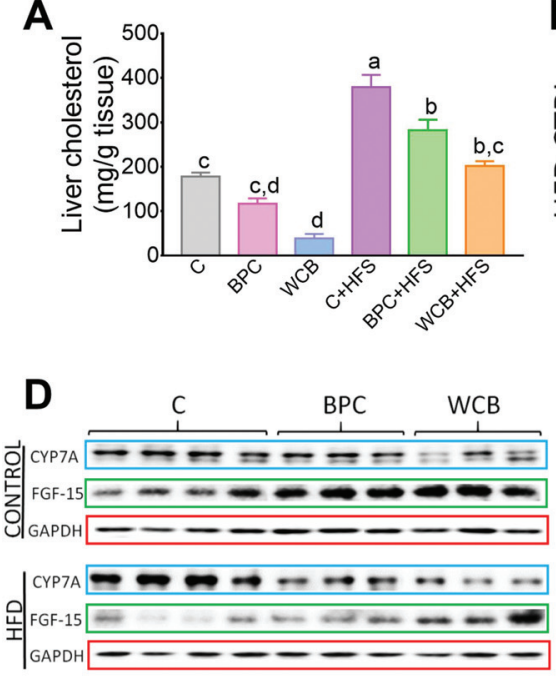

B

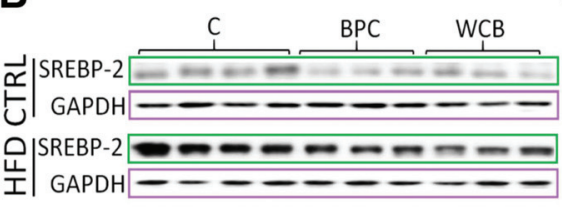

C

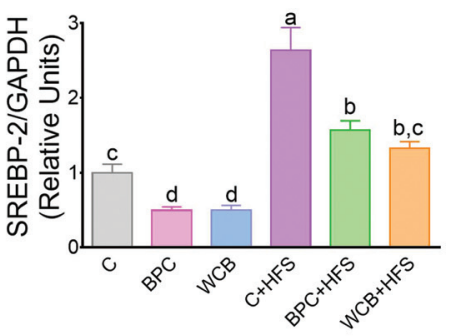

$\mathbf{F}$

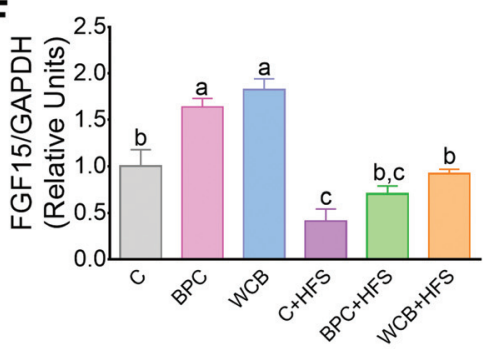

G

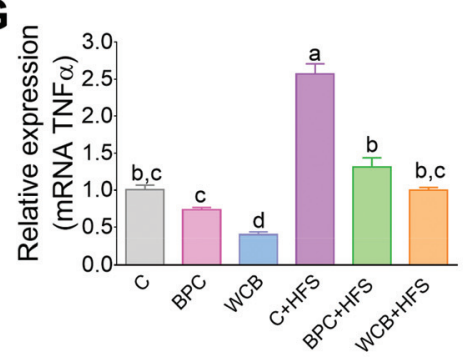

$\mathrm{H}$

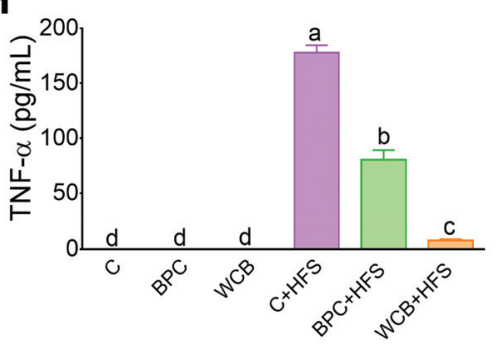

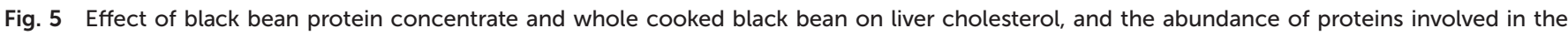

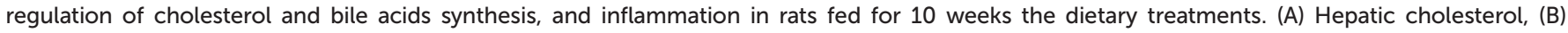

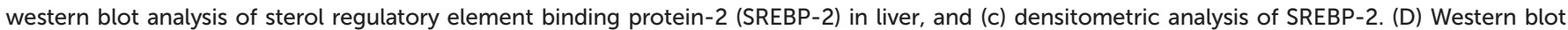

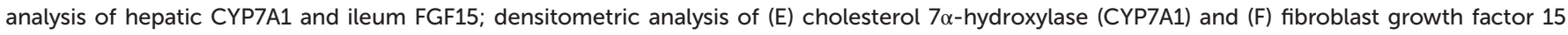

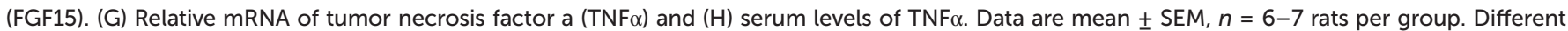
letters indicate significant differences among groups, $a>b>c>d, p<=0.05$. 
control group. These results were accompanied by a significant increase in the circulating levels of TNF- $\alpha$. Consumption of BPC + HFS or WCB + HFS, significantly reduced the liver mRNA abundance of TNF- $\alpha$ by $49 \%$ and $61.1 \%$ respectively compared to the HFS group (Fig. 5G). This was accompanied by a reduction in serum $\mathrm{TNF} \alpha$ concentration after the consumption of BPC + HFS or WCB + HFS compared to the $\mathrm{C}+$ HFS groups (Fig. 5H).

\section{Discussion}

In Mexico, as in the rest of Mesoamerica, beans have been the preferred legume for centuries. ${ }^{27}$ However, over the past 30 years, the eating habits of most segments of the population have shifted to a western-style diet containing refined sugars and more fat, in the form of processed foods. ${ }^{28}$ However, despite several government interventions, the overweight and obesity pandemic is still ongoing. ${ }^{29}$ More in-depth nutritional education is needed, and an interesting alternative would be to improve the formulations of processed foods so that they have a better impact and healthier options. The use of new bean preparations such as BPC or WPC are interesting raw materials from a food processing point of view because of the health benefits they can bring to the consumer. In this study, we evaluated an experimental black bean protein concentrate that could be used as a useful protein for the re-formulation of processed foods and was compared with the whole cooked black bean preparation containing all the components of whole bean grains.

It has been suggested that the quality of legume proteins is not optimal when compared to a protein of animal origin. However, body composition after consumption of black bean protein and its metabolic consequences have never been evaluated. The results were very interesting because animals fed bean protein, with and without fat, gained less weight than those fed casein. One might think that this was due to the quality of the protein, however, when body composition was measured, the results showed that this was due to a decrease in body fat, and that the percentage of lean mass was equal to that of animals fed animal protein, indicating that the amino acid pattern of black beans is sufficient to maintain adequate lean mass, as well as those fed animal protein. The results of the present study demonstrated that the consumption of BPC prevents body weight gain even after the consumption of a high fat diet. These results suggest that the black bean protein is a good alternative to be included in the formulation of foods to attenuate body weight and fat gain. These effects could be in part due to an increase in energy expenditure. Interestingly the presence of resistant starch in the whole cooked black bean were more effective to decrease serum triglycerides and total cholesterol, however BPC or WCB significantly decreased total cholesterol and triglycerides in the high fat diet. Due to the low-medium glycemic index of the black bean, ${ }^{30}$ the serum glucose and insulin concentration, as well as the glucose tolerance test were similar to the control group, effect that was more evident after the consumption of a high diet. These results could be explained in part due to an increase in fatty acid oxidation particularly in those fed a high fat diet and a decrease in liver lipogenesis.

During the development of obesity in animal models and in humans, one of the alterations highly frequently observed in the presence of hepatic steatosis. ${ }^{31}$ The accumulation in liver of triglycerides and cholesterol can produce lipotoxicity, and in some instances can progress into steatohepatitis and liver fibrosis. ${ }^{32}$ Interestingly, in the present study, the results showed particularly in animals fed a high fat diet, that the consumption of black bean, either as BPC or WCB decreased hepatic steatosis associated with a decrease in the concentration of triglycerides and cholesterol. The modulation of the lipogenic and fatty acid oxidation genes were more effectively regulated by BPC associated with an adequate insulin sensitivity.

It is important to point out that the amino acid profile present in the black bean concentrate may modulate serum insulin levels. Previous reports have shown that black beans contain anthocyanins ${ }^{33}$ and phenolic compounds that may also improve the insulin sensitivity. ${ }^{34}$ Particularly, the phenolic compounds and flavonoids present in black beans are mainly delphinidin 3-glucoside, petunidin 3-glucoside, and malvidin 3-glucoside. In our study, the bean protein concentrate maintained about $75 \%$ of the original phenolic content of whole cooked beans (WCB) $(1.85 \pm 0.08$ vs. $2.42 \pm 0.08 \mathrm{mg}$ gallic acid per $\mathrm{g}$ sample) after the concentration process, and 10 times less resistant starch than that of whole cooked beans (WCB) (Table 3). BPC showed a beneficial effect that can be explained in part by the fact that its flavonoid content was twice the value shown by WCB, reaching values of about $2 \mathrm{mg} \mathrm{g}^{-1}$ BPC due to the concentration process (Table 3 ). In addition, anthocyanins are a type of flavonoid compounds with antioxidant capacity that can promote some effects preventing oxidative stress.

Interestingly, it has been previously demonstrated that a methanol extract from black bean seed coats contain quercetin 3-o-glucoside and soyasaponin-Af as the primary flavonoid and saponin. Interestingly, ingestion of this extract reduced hepatic cholesterol, in part, via bile acid synthesis. ${ }^{35}$ In the present study, the results showed that black bean consumption decreased the hepatic concentration of cholesterol. In fact, our results suggest that the mechanism by which consumption of black bean decreased liver cholesterol could be, at least in part, by decreasing the expression of SREBP-2, a transcription factor that regulates the expression of genes of cholesterol synthesis. Furthermore, the reduction of hepatic cholesterol was accompanied by a reduction in the expression of Cyp7A1, an enzyme that is the rate-limiting step in the formation of bile acids by the classical pathway. The decrease in the expression of Cyp7A1 was also in part reinforced by an increase in the protein abundance of FGF15 in the ileum, a protein that is normally synthesized in response to an elevated concentration of intestinal bile acids, ${ }^{25}$ however, recent evidence suggest that the gut microbiota can modify the bile acid-FXR-FGF15 axis, 
and it has been demonstrated that phenolic compounds can interact and modify the gut microbiota. ${ }^{36}$ Interestingly, it has been demonstrated lately that a preparation of black bean can selectively modify the gut microbiota, ${ }^{30}$ therefore studies are needed to establish a potential interaction between the BPC, the gut microbiota and the mechanism involving FGF15 in order to regulate hepatic cholesterol concentration.

\section{Conclusions}

The present study demonstrated that the long-term consumption for 10 weeks of BPC is a good alternative to prevent body weight and fat gain, leading in several beneficial effects on serum lipids and an improvement in glucose metabolism. Interestingly, several of the effects were similar with those observed with WCB, a preparation containing more of the components of raw black beans. Importantly, the consumption of BPC significantly reduced the accumulation of lipids in the liver, including either triglyceride and cholesterol, even in rats fed a high-fat diet. The results obtained suggest that daily consumption of black beans can regulate the postprandial metabolic response, thus contributing to maintaining good metabolic health by counteracting the effects associated with the development of obesity. In addition, the use of BPC is a recommended preparation that can be used in the development of new dietary strategies, including the development of new products as part of a dietary strategy to produce health benefits in obese subjects, or those with type 2 diabetes.

\section{Ethical statement}

All animal procedures were performed in accordance with the Guidelines for Care and Use of Laboratory Animals of the Instituto Nacional de Ciencias Medicas y Nutrición Salvador Zubirán (INCMNSZ) Mexico City and approved by the Animal Ethics Committee of INCMNSZ with the protocol code FNU-1744-16/18-1.

\section{Conflicts of interest}

There are no conflicts of interest to declare.

\section{Acknowledgements}

The authors thank Luzallie Jasso for the analysis of trypsininhibitor activities, Perla Osorio for resistant starch analyses, and José Antonio Bojorge, Campo Verde SA de CV for the donation of black bean.

This project was partly financed by UNAM-PAIP-FQ 50009096 and by the Instituto Nacional de Ciencias Medicas y Nutricion Salvador Zubiran. Irma Hernandez received a scholarship from CONACYT. CONABIO financed the costs of publication trough Project 9380 GEF Agrobiodiversidad Mexicana.

\section{References}

1 C. J. Rebello, F. L. Greenway and J. W. Finley, A review of the nutritional value of legumes and their effects on obesity and its related co-morbidities, Obes. Rev., 2014, 15, 392-407.

2 R. Campos-Vega, R. Reynoso-Camacho, G. Pedraza-Aboytes, J. A. Acosta-Gallegos, S. H. Guzman-Maldonado, O. Paredes-Lopez, B. D. Oomah and G. Loarca-Pina, Chemical composition and in vitro polysaccharide fermentation of different beans (Phaseolus vulgaris L.), J. Food Sci., 2009, 74, T59-T65.

3 A. D. T. Fabbri, R. W. Schacht and G. A. Crosby, Evaluation of resistant starch content of cooked black beans, pinto beans, and chickpeas, NFS J., 2016, 3, 8-12.

4 C. Reyes-Moreno and O. Paredes-Lopez, Hard-to-cook phenomenon in common beans (Phaseolus vulgaris) - A review, Crit. Rev. Food Sci. Nutr., 1993, 33, 224-286.

5 H. M. Sanchez-Arteaga, J. E. Urias-Silvas, H. EspinosaAndrews and E. Garcia-Marquez, Effect of chemical composition and thermal properties on the cooking quality of common beans (Phaseolus vulgaris), CyTA - J. Food, 2014, 13, 385-391.

6 O. B. Tandon, R. Bressani, N. S. Scrimshaw and F. Le Beau, Nutritive value of beans. Nutrients in Central American beans, J. Agric. Food Chem., 1957, 5, 137-142.

7 A. P. Nugent, Health properties of resistant starch, Nutr. Bull., 2005, 30, 27-54.

8 P. Osorio-Díaz, L. A. Bello-Perez, S. G. Sayago-Ayerdi, M. P. Benitez-Reyes, J. Tovar and O. Paredes-Lopez, Effect of processing and storage time on in vitro digestibility and resistant starch content of two bean (Phaseolus vulgaris L) varieties, J. Sci. Food Agric., 2003, 83, 1283-1288.

9 L. Diaz-Batalla, J. M. Widholm, G. C. Fahey Jr., E. CastanoTostado and O. Paredes-Lopez, Chemical components with health implications in wild and cultivated Mexican common bean seeds (Phaseolus vulgaris L.), J. Agric. Food Chem., 2006, 54, 2045-2052.

10 L. Mojica, M. Berhow and E. Gonzalez de Mejia, Black bean anthocyanin-rich extracts as food colorants: Physicochemical stability and antidiabetes potential, Food Chem., 2017, 229, 628-639.

11 H. Li, J. Li, Y. Shen, J. Wang and D. Zhou, Legume Consumption and All-Cause and Cardiovascular Disease Mortality, BioMed Res. Int., 2017, 2017, 8450618.

12 L. A. Bazzano, A. M. Thompson, M. T. Tees, C. H. Nguyen and D. M. Winham, Non-soy legume consumption lowers cholesterol levels: a meta-analysis of randomized controlled trials, Nutr., Metab. Cardiovasc. Dis., 2011, 21, 94-103.

13 E. J. Reverri, J. M. Randolph, F. M. Steinberg, C. T. Kappagoda, I. Edirisinghe and B. M. Burton-Freeman, Black Beans, Fiber, and Antioxidant Capacity Pilot Study: Examination of Whole Foods vs. Functional Components on Postprandial Metabolic, Oxidative Stress, and Inflammation in Adults with Metabolic Syndrome, Nutrients, 2015, 7, 6139-6154. 
14 D. E. Kelley, B. H. Goodpaster and L. Storlien, Muscle triglyceride and insulin resistance, Annu. Rev. Nutr., 2002, 22, 325-346.

15 D. M. Muoio and C. B. Newgard, Mechanisms of disease: Molecular and metabolic mechanisms of insulin resistance and beta-cell failure in type 2 diabetes, Nat. Rev. Mol. Cell Biol., 2008, 9, 193-205.

16 L. Azadbakht, F. Haghighatdoost and A. Esmaillzadeh, Legumes: A component of a healthy diet, J. Res. Med. Sci., 2011, 16, 121-122.

17 M. L. Kakade, J. J. Rackis, J. E. McGhee and G. Puski, Determination of trypsin inhibitor activity of soy products: a collaborative analysis of an improved procedure, Cereal Chem., 1974, 51, 376-381.

18 S. Klomklao, S. Benjakul, H. Kishimura and M. Chaijan, Extraction, purification and properties of trypsin inhibitor from Thai mung bean (Vigna radiata (L.) R. Wilczek), Food Chem., 2011, 129, 1348-1354.

19 P. G. Reeves, F. H. Nielsen and G. C. Fahey Jr., AIN-93 purified diets for laboratory rodents: final report of the American Institute of Nutrition ad hoc writing committee on the reformulation of the AIN-76A rodent diet, J. Nutr., 1993, 123, 1939-1951.

20 G. Aleman, A. L. Castro, A. Vigil-Martinez, I. TorreVillalvazo, A. Diaz-Villasenor, L. G. Noriega, I. Medina-Vera, G. Ordaz, N. Torres and A. R. Tovar, Interaction between the amount of dietary protein and the environmental temperature on the expression of browning markers in adipose tissue of rats, Genes Nutr., 2019, 14, 19.

21 M. Sanchez-Tapia, A. W. Miller, O. Granados-Portillo, A. R. Tovar and N. Torres, The development of metabolic endotoxemia is dependent on the type of sweetener and the presence of saturated fat in the diet, Gut Microbes, 2020, 12, 1801301.

22 J. Folch, M. Lees and G. H. Sloane Stanley, A simple method for the isolation and purification of total lipides from animal tissues, J. Biol. Chem., 1957, 226, 497509.

23 P. Chomczynski and N. Sacchi, Single-step method of RNA isolation by acid guanidinium thiocyanate-phenol-chloroform extraction, Anal. Biochem., 1987, 162, 156-159.

24 M. W. Pfaffl, A new mathematical model for relative quantification in real-time RT-PCR, Nucleic Acids Res., 2001, 29, e45.

25 S. A. Kliewer and D. J. Mangelsdorf, Bile Acids as Hormones: The FXR-FGF15/19 Pathway, Dig. Dis., 2015, 33, 327-331.

26 G. S. Hotamisligil, N. S. Shargill and B. M. Spiegelman, Adipose expression of tumor necrosis factor-alpha: direct role in obesity-linked insulin resistance, Science, 1993, 259, 87-91.

27 E. Bitocchi, L. Nanni, E. Bellucci, M. Rossi, A. Giardini, P. S. Zeuli, G. Logozzo, J. Stougaard, P. McClean, G. Attene and R. Papa, Mesoamerican origin of the common bean (Phaseolus vulgaris L.) is revealed by sequence data, Proc. Natl. Acad. Sci. U. S. A., 2012, 109, E788-E796.

28 B. M. Popkin, L. S. Adair and S. W. Ng, Global nutrition transition and the pandemic of obesity in developing countries, Nutr. Rev., 2012, 70, 3-21.

29 M. Hernandez-Avila, J. Rivera-Dommarco, T. Shamah Levy, L. Cuevas Nasu, L. M. Gomez Acosta, E. B. Gaona Pineda, and M. Romero Martinez, Encuesta Nacional de Salud y Nutrición de Medio Camino 2016, INSP, Mexico, 2016.

30 M. Sanchez-Tapia, I. Hernandez-Velazquez, E. PichardoOntiveros, O. Granados-Portillo, A. Galvez, A. R. Tovar and N. Torres, Consumption of Cooked Black Beans Stimulates a Cluster of Some Clostridia Class Bacteria Decreasing Inflammatory Response and Improving Insulin Sensitivity, Nutrients, 2020, 12, 1182.

31 E. Fabbrini, S. Sullivan and S. Klein, Obesity and nonalcoholic fatty liver disease: biochemical, metabolic, and clinical implications, Hepatology, 2010, 51, 679-689.

32 B. A. Neuschwander-Tetri, Hepatic lipotoxicity and the pathogenesis of nonalcoholic steatohepatitis: the central role of nontriglyceride fatty acid metabolites, Hepatology, 2010, 52, 774-788.

33 K. Yoshida, Y. Sato, R. Okuno, K. Kameda, M. Isobe and T. Kondo, Structural Analysis and Measurement of Anthocyanins from Colored Seed Coats of Vigna, Phaseolus, and Glycine Legumes, Biosci., Biotechnol., Biochem., 1995, 60, 589-593.

34 K. Damian-Medina, Y. Salinas-Moreno, D. Milenkovic, L. Figueroa-Yanez, E. Marino-Marmolejo, I. HigueraCiapara, A. Vallejo-Cardona and E. Lugo-Cervantes, In silico analysis of antidiabetic potential of phenolic compounds from blue corn (Zea mays L.) and black bean (Phaseolus vulgaris L.), Heliyon, 2020, 6, e03632.

35 R. A. Chavez-Santoscoy, J. A. Gutierrez-Uribe, O. Granados, I. Torre-Villalvazo, S. O. Serna-Saldivar, N. Torres, B. Palacios-Gonzalez and A. R. Tovar, Flavonoids and saponins extracted from black bean (Phaseolus vulgaris L.) seed coats modulate lipid metabolism and biliary cholesterol secretion in C57BL/6 mice, Br. J. Nutr., 2014, 112, 886-899.

36 A. Kumar Singh, C. Cabral, R. Kumar, R. Ganguly, H. Kumar Rana, A. Gupta, M. Rosaria Lauro, C. Carbone, F. Reis and A. K. Pandey, Beneficial Effects of Dietary Polyphenols on Gut Microbiota and Strategies to Improve Delivery Efficiency, Nutrients, 2019, 11, 2216. 